\title{
CHARACTERIZING SHAPES OF COMPACTA
}

\author{
BY T. A. CHAPMAN ${ }^{1}$ \\ Communicated by Steve Armentrout, October 18, 1971
}

\begin{abstract}
In this note we announce a characterization of Borsuk's concept of shape for finite-dimensional compact metric spaces. Let $X, Y$ be compact subsets of some Euclidean space $E^{n}$ such that (1) $X$ lies in some Euclidean subspace of $E^{n}$ having codimension at least $2 \operatorname{dim} X+1$ and (2) $Y$ lies in some Euclidean subspace of $E^{n}$ having codimension at least $2 \operatorname{dim} Y+1$. Then $n$ can be chosen large enough (and dependent only on $\operatorname{dim} X, \operatorname{dim} Y$ ) such that the following is true: $X$ and $Y$ have the same shape iff $E^{n} \backslash X$ and $E^{n} \backslash Y$ are homeomorphic. We also discuss the relationship of this result to previously known characterizations of shape.
\end{abstract}

1. Introduction. The objective of this note is to announce a characterization of shape (as introduced by Borsuk in [4]) for finite-dimensional compact metric spaces in terms of the homeomorphism types of the complements under stable embeddings in large-dimensional Euclidean spaces. The precise statement is given in $\$ 4$ and the details of its proof will appear elsewhere [9]. This result confirms an informal conversational conjecture of Morton Brown concerning the geometric intuition about what shape ought to mean. It and a companion earlier result of the author [6] cited in $\$ 3$ below give easily stated characterizations of shape. They also put the study of shape in the point-set (homeomorphism) domain as distinct from the homotopy domain of the definition of Borsuk and of the characterization of Mardesic and Segal cited in $\$ 3$ below.

In $\$ 2$ we make some intuitive comments concerning shapes of compact metric spaces (compacta) without actually giving any definitions. This section is intended only for those readers not familiar with shape theory. In $\$ 3$ we cite some other instances in which shapes of compacta have been characterized by using approaches quite different from that of Borsuk who views shape as a generalization of homotopy type to nonANR's (metric). The notation

$$
\operatorname{Sh}(X)=\operatorname{Sh}(Y)
$$

will be used to indicate that compacta $X$ and $Y$ have the same shape.

2. Intuitive shape theory. The term shape theory actually refers to an entire category of objects (compacta) and morphisms (which we shall not specify), but we are only interested here in the equivalence relation

AMS 1969 subject classifications. Primary 5540; Secondary 5478.

Key words and phrases. Shape, ANR, Hilbert space, Hilbert cube.

${ }^{1}$ Supported in part by NSF Grant GP-28374.

Copyright (C) American Mathematical Society 1972 
$\operatorname{Sh}(X)=\operatorname{Sh}(Y)$ (for compacta $X$ and $Y$ ) which one introduces into this category in much the same way that homotopy equivalence is introduced into the category of topological spaces and maps.

Roughly speaking this concept enables one to compare global properties of compacta, while virtually ignoring their local properties. For example the pseudo-arc has the shape of an arc. Also the Warsaw circle has the shape of a circle, where by the Warsaw circle we mean the compactum in $E^{2}$ which is the union of the compactum

$$
A=(\{0\} \times[-1,1]) \cup\{(x, \sin (1 / x)) \mid 0<x \leqq 2 / \pi\}
$$

and an arc which has endpoints $(0,0),(2 / \pi, 1)$ and which intersects $A$ only in these points [5].

If $X$ and $Y$ are compacta which are homotopy equivalent, then $\operatorname{Sh}(X)$ $=\operatorname{Sh}(Y)$. Also if $X$ and $Y$ are compact ANR's, then $X$ and $Y$ are homotopy equivalent iff $\operatorname{Sh}(X)=\operatorname{Sh}(Y)$ [4]. Thus if compacta have the same shape and sufficient local structure, then they are also homotopy equivalent. In the above paragraph we have cited two examples of compacta which have the same shape but which are not homotopy equivalent.

In order to define what it means for compacta $X$ and $Y$ to have the same shape (using Borsuk's approach [4]), one considers $X$ and $Y$ as subsets of Hilbert space $l_{2}$ (or the Hilbert cube $Q$ ) and then requires that there exist sequences of approximative maps of $X$ towards $Y$ and $Y$ towards $X$ which satisfy certain homotopy properties and are defined on appropriate (systems of) neighborhoods of these sets. We shall not be more precise than this and we only remark (as in \$1) that this definition succeeds in generalizing the notion of homotopy equivalence to nonARN's. Using this approach Borsuk and a number of colleagues and students have been able to obtain analogues of many fundamental concepts of homotopy theory. Most of the results appear in Fundamenta Mathematicae or the Bulletin of the Polish Academy of Sciences.

3. Alternate approaches to shape. In [13] Mardesic and Segal introduced an alternate approach to shape theory which, like the author's, is equivalent to Borsuk's. Roughly their idea is to consider compacta represented as inverse systems of compact ANR's. They replace the approximative maps of Borsuk by maps between inverse systems, where the usual commutativity of diagrams is replaced by homotopy commutativity. In this approach compacta need not be considered in terms of embeddings in Hilbert space or the Hilbert cube, and there is complete freedom in the choice of such expansions of a given compactum. It is not surprising that such a substantially different viewpoint of shape theory has proven quite effective in dealing with shape problems (see [12] for one of a number of papers on the subject). 
In [10] D. W. Henderson used some recent results in the topology of infinite-dimensional Hilbert manifolds to obtain some results in shape theory. In particular he characterized those compacta which have the shape of a point by showing that a compactum $X \subset l_{2}$ satisfies $\operatorname{Sh}(X)$ $=\operatorname{Sh}(\{$ point $\})$ iff the quotient space $l_{2} / X$ is homeomorphic to $l_{2}$ (where $l_{2} / X$ is the quotient space of $l_{2}$ by the decomposition whose only nontrivial element is $X$ ).

In [6] the author used a different infinite-dimensional approach to obtain necessary and sufficient conditions in order for two compacta to have the same shape. Since this characterization is similar to (and to some extent motivates) the finite-dimensional characterization cited in $\$ 4$ below, we state it. Let the Hilbert cube $Q$ be represented by $\prod_{i=1}^{\infty} I_{i}$, where each $I_{i}$ is the closed interval $[0,1]$, and let $s$ denote the pseudo-interior of $Q$, i.e., $s=\prod_{i=1}^{\infty} I_{i}^{o}$, where each $I_{i}^{o}$ is the open interval $(0,1)$. The main result of [6] is a follows:

If $X, Y \subset s$ are compacta, then $\operatorname{Sh}(X)=\operatorname{Sh}(Y)$ iff $Q \backslash X$ and $Q \backslash Y$ are homeomorphic.

We remark that a condition like $X, Y \subset s$ is crucial and in general cannot be replaced by the weaker condition $X, Y \subset Q$. Also it follows from [1] that for any compacta $X, Y \subset s, s \backslash X$ and $s \backslash Y$ are always homeomorphic. For possible easier geometric intuition one may replace the condition $X, Y \subset s$ by the known equivalent condition $X, Y \subset W$, where $W=\left\{\left(x_{i}\right) \in Q \mid x_{1}=1\right\}$.

Using this characterization and some basic techniques from infinitedimensional topology, it is possible to obtain some results in pure shape theory apparently not accessible from the other theories as well as to give alternative (and shorter) proofs of several of the basic theorems (see [2], [7], and [8]).

4. Statements of results. For the finite-dimensional characterization we first need a definition. A compactum $X$ in Euclidean space $E^{n}$ is said to be stably embedded provided that $X$ lies in some Euclidean subspace of $E^{n}$ having codimension at least $2 \operatorname{dim} X+1$. It is easy to see that if $X$ is stably embedded in $E^{n}$ and if $E^{n}$ is then identified with some Euclidean subspace of $E^{m}$, then $X$ is also stably embedded in $E^{m}$. Using a result of Klee [11] it follows that if $X$ is a compactum and $X^{\prime}, X^{\prime \prime} \subset E^{n}$ are stably embedded copies of $X$, then there exists a homeomorphism of $E^{n}$ onto itself taking $X^{\prime}$ onto $X^{\prime \prime}$. Thus all stably embedded copies of a given compactum in an Euclidean space are equivalent.

THEOREM [9]. For each integer $m>0$ there exists an integer $n(m)>0$ such that the following property is satisfied: If $X, Y \subset E^{n}$ are stably embedded compacta such that $\operatorname{dim} X, \operatorname{dim} Y \leqq m$ and $n \geqq n(m)$, then $\operatorname{Sh}(X)$ $=\operatorname{Sh}(Y)$ iff $E^{n} \backslash X$ and $E^{n} \backslash Y$ are homeomorphic. 
Moreover in the only if part of the Theorem, the homeomorphism can be constructed to be the identify off of any topological $n$-cell containing $X \cup Y$ in its interior. We also remark that we can always choose

$$
n(m)=3 m+3,
$$

but this number is the result of several calculations and can very probably be reduced.

In the proof of the infinite-dimensional characterization of [6], cited in $\$ 3$ above, some recent results in infinite-dimensional manifolds modeled on $Q$ were used. The proof of the Theorem above (as given in [9]) has the same broad outline as the proof in [6], but the details of the argument are largely different because of the lack of suitable finite-dimensional analogues of certain infinite-dimensional techniques.

In an analogous fashion one can define what it means for a compactum to be stably embedded in the $n$-sphere $S^{n}$ and obtain the following result directly from the Theorem.

COROLlary 1. For each integer $m>0$ there exists an integer $n(m)>0$ such that the following property is satisfied: If $X, Y \subset S^{n}$ are stably embedded compacta such that $\operatorname{dim} X, \operatorname{dim} Y \leqq m$ and $n \geqq n(m)$, then $\operatorname{Sh}(X)=$ $\operatorname{Sh}(Y)$ iff $S^{n} \backslash X$ and $S^{n} \backslash Y$ are homeomorphic.

In the Theorem above it should be noted that merely assuming the codimension to be large will not generally suffice. For example in any Euclidean space $E^{n}, n \geqq 3$, Blankinship [3] has constructed arcs whose complements are not simply connected, and therefore cannot be homeomorphic to the complement of any stably embedded arc. However if the compacta are polyhedra, then the wildness exhibited by the Blankinship examples cannot occur, and a largeness condition on the codimension will alone suffice. But even in such a nice situation the largeness condition on the codimension is essential, for Henderson [10] has constructed 2-dimensional compact polyhedra $X, Y \subset E^{3}$ of different shape for which $E^{3} \backslash X$ and $E^{3} \backslash Y$ are homeomorphic.

The following result is a corollary of the proof of the Theorem above, although it is possible to give a shorter more direct proof without even defining the notion of shape (as shape and homotopy type agree on compact ANR's, and therefore on compact polyhedra).

COROLLARY 2. For each integer $m>0$ there exists an integer $n(m)>0$ such that the following property is satisfied: If $X, Y \subset E^{n}$ are compact polyhedra such that $\operatorname{dim} X, \operatorname{dim} Y \leqq m$ and $n \geqq n(m)$, then $X$ and $Y$ are homotopy equivalent iff $E^{n} \backslash X$ and $E^{n} \backslash Y$ are homeomorphic.

ADDED IN PROOF. In a forthcoming paper, Concerning the shapes of finite-dimensional compacta, R. Geoghegan and R. Summerhill have obtained a result which generalizes the above Theorem. 


\section{REFERENCES}

1. R. D. Anderson, Topological properties of the Hilbert cube and the infinite product of open intervals, Trans. Amer. Math. Soc. 126 (1967), 200-216. MR 34 \# 5045.

2. - Point-like decompositions of the Hilbert cube (in preparation).

3. W. A. Blankinship, Generalization of a construction of Antoine, Ann. of Math. (2) 53 (1951), 276-297. MR 12, 730.

4. K. Borsuk, Concerning homotopy properties of compacta, Fund. Math. 62 (1968), 223-254. MR 37 \# 4811.

5. - Concerning the notion of the shape of compacts, Proc. Internat. Sympos. on Topology and its Applications, Herceg Novi, 1968.

6. T. A. Chapman, On some applications of infinite-dimensional manifolds to the theory of shape, Fund. Math. (to appear).

7. - Some results on shape and fundamental absolute retracts, Bull. Acad. Polon. (to appear).

8. hapes of some decomposition spaces, Bull. Acad. Polon. (to appear).

9. Shapes of finite-dimensional compacta, Fund. Math. (to appear).

10. D. W. Henderson, Applications of finite-dimensional manifolds to quotient spaces of complete ANR's, Bull. Acad. Polon. (to appear).

11. V. L. Klee, Some topological properties of convex sets, Trans. Amer. Math. Soc. 78 (1955), 30-45. MR 16, 1030.

12. S. Mardesic, On the shape of the quotient space $S^{n} / A$, Bull. Acad. Polon. 19 (1971).

13. S. Mardesic and J. Segal, Shapes of compacta and ANR-systems, Fund. Math. 72 (1971), 57-75.

Mathematical Center, Amsterdam, The Netherlands

DePartment of Mathematics, University of Kentucky, Lexington, Kentucky 40506 (current address) 